\title{
Histochemical Changes in Rat Sperm Malate Dehydrogenase Activity during Passage through Epididymis
}

\author{
TOKIHIRo MATSUZAWA AND Hiroshi SAWADA \\ Laboratory of Biology, Obihiro University of Agriculture and Veterinary \\ Medicine, Inada-cho, Obihiro, Hokkaido 080, Japan
}

\begin{abstract}
Histochemical activity of malate dehydrogenase (MDH: E. C. 1.1.1.37) in rat sperm mitochondria decreased during the epididymal transit of sperm. In an electrophoretic study two major MDH isozymes (MDH-A and MDH-B) were demonstrated in the sperm. The epididymal sperm showed two minor isozymes associated with MDH-A, while the testicular sperm did not.
\end{abstract}

Malate dehydrogenase (MDH: E. C. 1.1.1.37) which catalyzes the reversible reaction from malate to oxaloacetate occurs in vertebrate tissues as two major classes of isozymes with different localization in extraparticulate cytoplasm and mitochondria (Thore et al., 1963; Kitto and Kaplan, 1966). Oxaloacetate is reduced by $\mathrm{MDH}$ with soluble $\mathrm{NADH}_{2}$ to form malate which enters the mitochondria again being oxidized by the enzyme with mitochondria bound NAD. The oxaloacetate produced is released into the cytoplasm, so making a shuttle of these substrates between the cytoplasm and mitochondria (Kaplan, 1963). Through the shuttle, cytoplasmic and mitochondrial MDH play an important role in the regulation of the $\mathrm{NAD} / \mathrm{NADH}_{2}$ ratio which is critical in glycolysis in the cell (Evers and Kaplan, 1975).

It is well known that in mammalia spermatozoa changes in metabolism are seen together with morphological changes during passage through the epididymis (Bedford,

Received August 6, 1986
1975).

However no study had been done on the histochemical change in sperm $\mathrm{MDH}$ activity with reference to the alteration of MDH zymogram during the transit of sperm through the epididymis.

The purpose of the present study was to investigate whether the histochemical changes in sperm MDH activity occur during their epididymal transit in accordance with the alteration of the sperm MDH zymogram.

\section{Materials and Methods}

Adult rats of Wistar strain (80-90 day old) were used throughout the experiment. Testicular sperm together with fluid were collected from the rete testis using a vinyl tube $(0.2 \mathrm{~mm}$ inner diameter) by the method of Hamilton (1980). The epididymis was dissected and separated into caput, corpus, and cauda. Then epididymal sperm from these segments were gently squeezed by forceps into a cold phosphate buffere saline (PBS, pH 7.4). Testicular and epididymal sperm were collected by centrifugation at $740 \times \mathrm{g}$ for 10 minutes and washed twice in the PBS. Each 
pellet of sperm was resuspended in a suitable amount of phosphate buffer ( $\mathrm{pH}$ 7.4) containing $0.1 \%$ of Triton-X 100 , subjected to freeze-thawing twice and sonicated with an ultra sonic cell disruptor (Tomy Hand Sonic UR-20P, Tokyo) at $20 \mathrm{~W}$ for 5 minutes. After centrifugation of the disrupted spermatozoa at $8,200 \times \mathrm{g}$ for 5 minutes the supernatant obtained was employed for enzyme assay and for analysis of the zymogram.

For histochemical observation of sperm MDH activity, the washed sperm were spread out on coverslips to dry at room temperature. Then they were stained by the method of Barka and Anderson (1963).

The activity in the supernatant of the sperm homogenate was assayed in a total volume of $2.0 \mathrm{ml}$ containing $60 \mu \mathrm{M}$ of sodium malate, 1.0 $\mu \mathrm{M}$ of 2-(4-iodophenyl)-3-(4-nitrophenyl)-5phenyl-tetrazolium chloride (INT), $0.76 \mu \mathrm{M}$ of NAD, $5.0 \mu \mathrm{M}$ of $\mathrm{NaCN}, 0.14 \mu \mathrm{M}$ of phenazine methosulfate and an appropriate amount of the supernatant. The reaction was initiated by the addititon of the supernatant. After 1-hr incubation at $37^{\circ} \mathrm{C}, 2.0 \mathrm{ml}$ of trichloroacetic acid was added to the reaction mixture to stop the reaction. The formazan formed in the mixture was extracted with $2.0 \mathrm{ml}$ of ethylacetate to measure the optical density at $500 \mathrm{~nm}$.

For analysis of the MDH zymogram, disc gel-electrophoresis was carried out according to the method of Davis (1964). Microslab gel electrophoresis was also carried out using the same buffer system as in disc gel electrophoresis and $7.5 \%$ of gel with commercial equipment for mini-slab gel electrophoresis (Atto, SJ 1060 MSD). The zymogram of MDH was demonstrated by the method previously described (Matsuzawa, 1979). Relative activities of MDH isozymes were measured with a densitometer at $500 \mathrm{~nm}$. The protein concentration was measured by the method of Lowry et al. (1951), using bovine serum albumin as a standard.

\section{Results}

In smear preparations of toluidine blue stain, sperm from both testis and epididymis were more than $94 \%$ pure.

Histochemically the deposit of formazan indicating $\mathrm{MDH}$ activity was prominent in testicular spermatozoa. In testicular sperm the deposit was found on the entire mitochondria which was of a spiral structure (Fig. 1a), while in caput sperm the deposit appeared throughout the mitochondrial sheath with scattered foci of high density (Fig. 1b). In corpus sperm a small formazan deposit was observed through the sheath without foci (Fig. 1c). The cauda sperm showed trace of MDH activity (Fig. 1d).

In both micro-slab and disc gel electrophoresis, two major isozymes (MDH-A and -B) were demonstrated in epididymal spermatozoa as well as in testicular spermatozoa. There was a tendency to relative activity of MDH-B, which moved more slowly, to decrease gradually during epididymal transit of spermatozoa (Table 1). In disc gel electrophoresis two sub-bands of isozymes associated with MDH-A, which run faster, were identified as $\mathrm{MDH}-\mathrm{A}_{1}$ and $-\mathrm{A}_{2}$ according to their electrophoretic mobilities. Testicular sperm did not show these isozymes, but in epididymal sperm $\mathrm{MDH}-\mathrm{A}_{1}$ and $-\mathrm{A}_{2}$ were demonstrated (Fig. 2). During sperm passage through the epididymis the activity of $\mathrm{MDH}-\mathrm{A}_{2}$ became relatively higher than that of MDH- $\mathrm{A}_{1}$.

No significant difference was observed in the specific activity of the enzyme in testicular and epididymal spermatozoa, though there was a tendency for the activity to decrease during the epididymal passage of spermatozoa (Table 2).

\section{Discussion}

Vogelmayr and his co-workers have reported that the rate of $\mathrm{CO}_{2}$ evolution from glucose was higher in the testicular sperm than in the ejaculated sperm in ram (Vogelmayr et al., 1967). On the other hand, the rate of conversion of glucose into lactate was lower in the testicular sperm than in the ejaculated sperm (Vogelmayr et al., 1967). These data suggest that in the testi- 

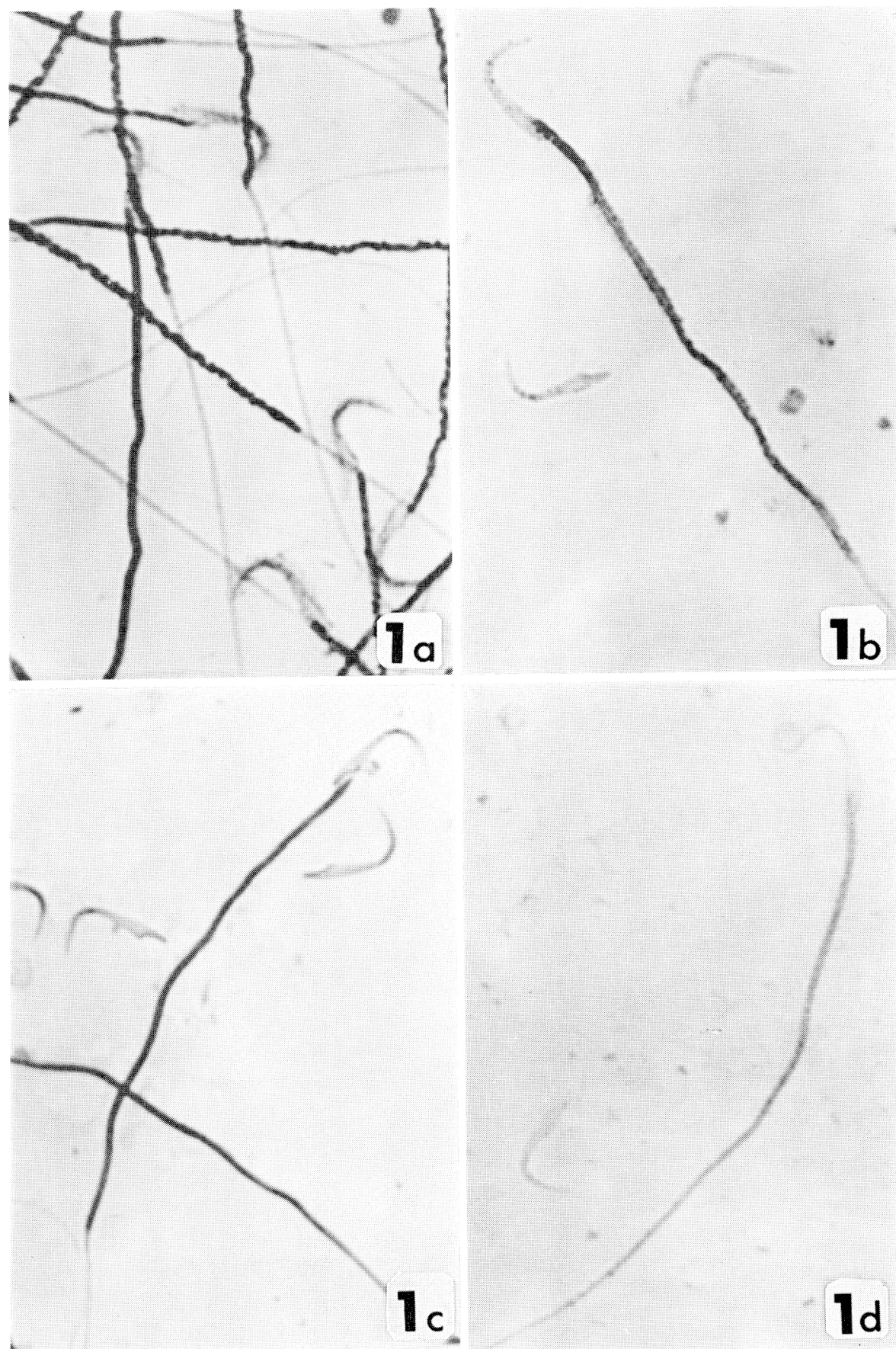

Fig. 1. Histochemical activity of rat sperm MDH $(\times 600)$.

a: Testicular sperm, b: Caput sperm, $\mathbf{c}$ : Corpus sperm, and $\mathbf{d}$ : Cauda sperm. 
Table 1. Relative activities (\%) of $\mathrm{MDH}$ isozymes in rat sperm

\begin{tabular}{ccccc}
\hline \hline Isozyme & Testicular sperm (7) & \multicolumn{3}{c}{ Epididymal sperm } \\
& & Caput (9) & Corpus (6) & Cauda (9) \\
\cline { 2 - 4 } A & $10.7 \pm 2.5$ & $13.9 \pm 2.9$ & $13.4 \pm 4.0$ & $14.3 \pm 4.7$ \\
B & $89.3 \pm 2.5$ & $86.1 \pm 3.2$ & $86.6 \pm 4.0$ & $85.7 \pm 3.3$ \\
\hline
\end{tabular}

Means \pm SD. Numbers in parentheses indicate the numbers of rats examined. The data shown were obtained by micro-slab gel electrophoresis.

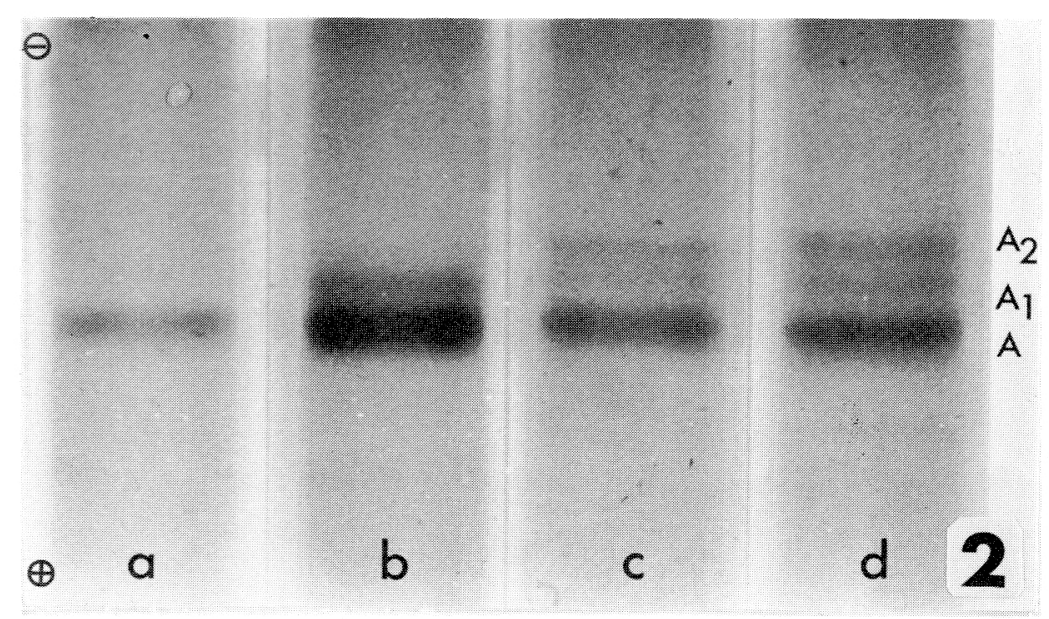

Fig. 2 Minor MDH isozymes which appeard in rat sperm.

a: Testicular sperm.

b: Caput sperm.

c: Corpus sperm.

d: Cauda sperm.

$\mathrm{A}_{1}$ and $\mathrm{A}_{2}$ indicate $\mathrm{MDH}-\mathrm{A}_{1}$ and $\mathrm{MDH}-$ $\mathrm{A}_{2}$, respectively.

Table 2. MDH activities of testicular and epididymal sperm ( $\mu \mathrm{g}$ of formazan produced $\cdot$ protein $\left.(\mu \mathrm{g})^{-1} \cdot \min ^{-1}\right)$

\begin{tabular}{cccc}
\hline Testicular & \multicolumn{3}{c}{ Epididymal sperm } \\
\cline { 2 - 4 } sperm (7) & Caput (9) & Corpus (6) & Cauda (7) \\
$0.21 \pm 0.05$ & $0.19 \pm 0.07$ & $0.18 \pm 0.07$ & $0.18 \pm 0.06$
\end{tabular}

Means $\pm S D$. Numbers in parentheses indicate numbers of rats examined.

cular sperm pyruvate produced via anaerobic glycolysis is easiy metabolized into acetyl CoA which is utilized through the citric acid cycle.

Hrudka (1979) has reported a strong activity of succinic dehydrogenase in the mitochondria of both rat and mouse sperm though the activity varied among individual spermatozoa. He also indicated that about $60 \%$ of ejaculated bovine sperm showed MDH activity. Succinic dehydrogenase activity was higher in rat testicular sperma- tozoa than in the cauda sperm (Matsuzawa, unpublished observation). Our histochemical observation showed that rat testicular sperm had higher $\mathrm{MDH}$ activity in the mitochondria than the epididymal sperm. These data indicate that mitochondria of testicular sperm are more active in energy metabolism than those of epididymal sperm.

The electrophoretic mobilities of MDH-A and MDH-B corresponded to those of cytoplasmic and mitochondrial MDH of hepatic cell origin. It is likely that MDH-A and MDH-B originate from the cytoplasm and mitochondria of the sperm, respectively. The histochemical observation and electrophoretic study suggested that the decrease in the sperm MDH-B to MDH-A ratio showed a decrease in the mitochondrial to cytoplasmic MDH ratio.

Conditions leading to decreased lipogenesis result in a decrease in the mito- 
chondrial to cytoplasmic $\mathrm{MDH}$ ratio (Thorne and Dent, 1970). Therefore, the decrease in the MDH-B to MDH-A ratio as shown by our data may indicate an increase in lipogenesis in rat sperm during the epididymal passage. Dacheux and Paquignon (1980) have reported that the incorportation of glucose into lipid decreases in the cauda sperm of boar. There may be a difference in the lipogenetic activity of spermatozoa according to the species.

In the present investigation two minor isozymes of $\mathrm{MDH}\left(\mathrm{MDH}-\mathrm{A}_{1}\right.$ and $\mathrm{MDH}-\mathrm{A}_{2}$ ) associated with MDH-A were observed in epididymal sperm. The appearance of these minor isozymes in epididymal sperm did not originate from other cells or epididymal fluid, because the cells of both testicular and epididymal sperm were highly pure. The physiological significance of these minor isozymes is unknown, though their appearance may be seen in connection with the decrease in histochemical MDH activity in the mitochondria.

The specific activity of sperm $\mathrm{MDH}$ did not change during the epididymal transit of sperm, though histochemical activity of MDH was higher in the testicular than in the epididymal sperm. This discrepancy may be explained as follows: during sperm passage through the epididymis a compensatory increase in cytosol $\mathrm{MDH}$ activity occurred in the sperm but mitochondrial MDH activity decreased.

\section{References}

Barka, T. and P. J. Anderson (1963). Oxidative enzyme. In: Histochemistry, Hoeber, New York. pp. 296-333.

Bedford, J. M. (1975). Maturation, transport, and fate of spermatozoa in the epididymis. In: Handbook of Physiology, Vol. 5, Sec. 7 (D. W. Hamilton and R. Creep eds.), American Physiological Society, Washington, D. C. pp. 303-317.

Dacheux, J. L. and M. Paquignon (1980). Ef- fects of caffein on ram and boar spermatozoa: Influence of their stage of maturation and the medium: Initiation of progressive motility of testicular spermatozoa. In: Testicular development, structure, and function (A. Steinberger and E. Steinberger eds.), Raven Press, New York. pp. 513-522.

Davis, B. J. (1964). Disc electrophoresis-II. Method and application of human serum protein. Ann. N. Y. Acad. Sci. 121, 404-427.

Everse, J. and N. O. Kaplan (1975). Mechanism of action and biological function of various dehydrogenase isoenzymes. In: Isozymes, Vol. 2, Physiological function (C. L. Markert ed.), Acad. Press, New York, San Francisco, and London. pp. 29-43.

Frudka, F. (1979). Cytochemistry of oxidoreductase in spermatozoa: The technique revisited. Andrologia 11, 337-357.

Hamilton, D. W. (1980). UDP-galactose : Nacetylglucosamine galactosyltransferase in fluid from rat rete testis and epididymis. Biol. Reprod. 23, 377-385.

Kaplan, N. O. (1963). Symposium on multiple forms of enzymes and control mechanisms. I. Multiple forms of enzymes. Bacteriol. Rev. 27, 155-169.

Kitto, G. B. and N. O. Kaplan (1966). Purification and properties of chicken heart mitochondrial and supernatant malic dehydrogenases. Biochemistry 5, 3966-3979.

Lowry, O. H., N. J. Rosebrough, A. L. Farr and R. J. Randall (1951). Protein measurement with Folin phenol reagent. J. Biol. Chem. 199, 265-275.

Matsuzawa, T. (1979). Staining specificity of histochemical reaction for $3 \alpha$-hydroxysteroid dehydrogenase activity in mouse testis. Zool. Mag. 88, 50-56.

Thorne, C. J. R. and N. J. Dent (1970). Malate dehydrogenase isoenzyme distribution in rat tissues. In: Enzyme and isozyme. Structure, properties, and function (D. Shugar ed.), Acad. Press, New York and London. pp. 87-99.

Thorne, C. J. R., L. I. Grossman and N. O. Kaplan (1963). Starch gel eleztrophoresis of malate dehydrogenase. Biochim. Biophys. Acta 73, 193-203.

Vogelmayr, J. K., T. W. Scott, B. P. Setchell and G. M. H. Waites (1967). Metabolism of testicular spermatozoa and characteristics of testicular fiuid collected from conscious ram. $J$. Reprod. Fert. 14, 87-99. 